Discussion Paper No. 04-50

\title{
The Impact of Equity-preferences
} on the Stability of

\section{Heterogeneous International Agreements}

\author{
Andreas Lange
}

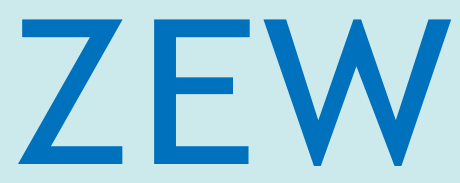

Zentrum für Europäische Wirtschaftsforschung $\mathrm{GmbH}$

Centre for European

Economic Research 


\title{
Discussion Paper No. 04-50 \\ The Impact of Equity-preferences on the Stability of Heterogeneous International Agreements
}

\author{
Andreas Lange
}

Download this ZEW Discussion Paper from our ftp server:

ftp://ftp.zew.de/pub/zew-docs/dp/dp0450.pdf

Die Discussion Papers dienen einer möglichst schnellen Verbreitung von neueren Forschungsarbeiten des ZEW. Die Beiträge liegen in alleiniger Verantwortung der Autoren und stellen nicht notwendigerweise die Meinung des ZEW dar.

Discussion Papers are intended to make results of ZEW research promptly available to other economists in order to encourage discussion and suggestions for revisions. The authors are solely responsible for the contents which do not necessarily represent the opinion of the ZEW. 


\section{Non-technical summary}

The solution of many environmental problems requires international cooperation. Due to the voluntary nature of cooperation, agreements must be beneficiary to all parties and be perceived to be equitable. Obligations in international agreements are typically differentiated between developing and developed countries. In particular in the climate debate, exemptions for developing countries are motivated by egalitarian arguments on per-capita emission levels. Besides this, possessory equity arguments to base targets on present or recent emission levels seem to play a vital role in several international agreements.

This paper studies the impact of equity considerations on the stability of international agreements between heterogeneous countries. We show that allowing countries to finance abatement projects in developing countries which, due to equity-reasons, have no binding emission targets can reduce the number of cooperating countries and thereby be welfare-decreasing. Equity-concerns in industrialized countries regarding the gap between per-capita emission levels of industrialized and developing countries lead to an increased reduction in industrialized countries but do not qualitatively change the incentives to cooperate. Only if countries are inequality-averse with respect to potential differences between their abatement targets and those of other industrialized countries is the inclusion of developing countries generally profitable both in terms of participation rates and of emission reduction. 


\title{
The impact of equity-preferences on the stability of heterogeneous international agreements
}

by

\author{
Andreas Lange \\ Centre for European Economic Research (ZEW), Mannheim \\ Interdisciplinary Institute for Environmental Economics, University of Heidelberg
}

\begin{abstract}
This paper studies the impact of equity considerations on the stability of international agreements between heterogeneous countries. We show that allowing countries to finance abatement projects in developing countries which, due to equity-reasons, have no binding emission targets can reduce the number of cooperating countries and thereby be welfare-decreasing. Equity-concerns in industrialized countries regarding the gap between per-capita emission levels of industrialized and developing countries lead to an increased reduction in industrialized countries but do not qualitatively change the incentives to cooperate. Only if countries are inequality-averse with respect to potential differences between their abatement targets and those of other industrialized countries is the inclusion of developing countries generally profitable both in terms of participation rates and of emission reduction.
\end{abstract}

JEL: C7, D63, H41, Q00

Keywords: international environmental negotiations, equity preference, coalition formation, per capita emission levels, inequality aversion

Correspondence: Centre for European Economic Research (ZEW), Postfach 103443 D68034 Mannheim, Germany, tel: +49 (0)621 1235-208, fax: -226, email: lange@zew.de

The author would like to thank Carsten Vogt, Tim Hoffmann, and Michael Finus for helpful comments. Funding of the research group "Institutionalization of International Negotiation Systems" and under grant LA $1333 / 2-1$ by the Deutsche Forschungsgemeinschaft (DFG) is gratefully acknowledged. 


\section{Introduction}

The solution of many environmental problems, such as global warming and the depletion of the ozone layer, requires international cooperation. Due to the voluntary nature of cooperation, agreements must be beneficiary to all parties and be perceived to be equitable. Obligations in international agreements are typically differentiated between developing and developed countries. In particular in the climate debate, exemptions for developing countries are motivated by egalitarian arguments on per-capita emission levels (Raymond 2003). Besides this, possessory equity arguments to base targets on present or recent emission levels seem to play a vital role in several international agreements. $^{1}$

This paper studies the impact of three different notions of equity on the chances of voluntary cooperation between countries: (i) Exempting developing countries from binding emission targets while opening them for other countries to finance abatement projects, (ii) equity-concerns with respect to differences in per-capita emissions between developing and developed countries, and (iii) inequality-aversion regarding the abatement targets of industrialized countries. We find that only the latter qualitatively increases the chances of cooperation.

The standard approach to the study of coalition formation was formulated by Barrett (1992, 1994) and Carraro and Siniscalco (1993). They use a two-period structure and study cooperation within a non-cooperative framework: Countries must first decide whether or not to join a coalition. Countries inside the coalition then behave cooperatively with respect to each other. However, both the coalition and the remaining countries choose their emission levels non-cooperatively. A coalition is stable if there is neither an incentive to join nor to leave the coalition. Barrett $(1992,1994,1997)$, Carraro and Siniscalco (1993), and Hoel (1992) have shown that - although there is co-

\footnotetext{
${ }^{1}$ Raymond (2003) characterizes grandfathering as a possessory rule. Equal measures for the signing countries are used in the LRTAP protocol on reduction of sulphur emissions (minus $30 \%$ based on 1980 level) or in the LRTAP protocol concerning the control of volatile organic compounds (minus $30 \%$ based on 1999 level).
} 
operation - the coalition size is rather small. Even if one allows for transfers to allocate the efficiency gains from cooperation according to a special rule (Nash-Bargaining or Shapley-value), as shown by Barrett $(1997,2001)$ and Botteon and Carraro (1997), only a few (three) countries cooperate. ${ }^{2}$ This pessimistic result on international cooperation is confirmed by empirical studies. Murdoch and Sandler (1997) on the Montreal protocol and Böhringer and Vogt (2004) on the Kyoto-protocol, find that those agreements do not substantially differ from symbolic policy.

All these theoretical studies base on a pure cost-benefit analysis and do not take any equity-considerations into account. Looking at the climate change debate, there are different dimensions in which equity considerations seem to play a vital role. Prominently, equity arguments are frequently used with respect to an equalization of per capita emission levels. ${ }^{3}$ They are stressed not only by delegates of developing countries, but also by environmental interest groups in developed countries. A government facing voters with such preferences must clearly take them into account. The weight that a government attaches to the equity argument will then depend on the impact these interest groups have on the national policy. As observed by Cazorla and Toman (2001, p. 238), "Equity might be one motivation for countries to pursue GHG emissions policies. However, equity principles will not override other elements of national self-interest."

In climate policy, equity issues play different roles: Reflecting the huge differences in living standards and per-capita emissions, developing countries are exempted from binding emission targets under the Kyoto-protocol. Other countries can however pursue abatement in developing countries by financing abatement projects under the Clean

\footnotetext{
${ }^{2}$ There is, however, the possibility of enlarging a coalition using an appropriate transfer scheme if countries are sufficiently asymmetric. Botteon and Carraro (1997) show that even the grand coalition can be stable if transfer payments are calculated using a two-stage Shapley-value procedure. Note that transfers are equivalent to an appropriate distribution of tradable emission permits.

${ }^{3}$ This principle of equity is even fixed in Article 3 of the Convention on Climate Change as well as in the decision approved by the COP 6 in Bonn which states that measures shall be implemented "...with a view to reducing emissions in a manner conducive to narrowing per capita differences between developed and developing country Parties".
} 
Development Mechanism. In addition, (some) industrialized countries may feel a certain responsibility for climate change since they have contributed the by far biggest share of greenhouse gas emissions. Evidence for this can be found in statements by environmental groups, which should have some impact as voters on the formulation of national policy. Therefore, this dimension of equity-concerns can be reflected by some "warm glow" effect from internalizing more than the own environmental damages or equivalently by inequality-aversion with respect to the differences in per-capita emissions between developing an developed countries. Another dimension of equity is concerned with a fair distribution of burdens which often comes down to similar reduction targets. Several proposal during the negotiations such as by the U.S. and Germany base the allocation of emission targets on present or recent emission levels (Raymond 2003,p.168-169). Similar abatement targets have the virtue of being easier to accept. On the one hand, they can provide a focal point in negotiations (Schelling 1960), on the other hand they might reduce political pressure: If a country has a much higher abatement target than most others, some political interest groups can use this comparison to lobby for less strict targets. If a country, however, has much smaller abatement targets than others, environmental interest groups probably more efficiently lobby in favor of a stricter environmental policy. Consequently, countries may exhibit inequality aversion with respect to differences in abatement targets of industrialized countries.

While the impact of equity considerations on financial burdens has been studied in several models (eg. Tol (2000), Böhringer and Helm (2001)), the importance of fairness and equity considerations - or equity-preferences - so far has played little role in the theoretical analysis of coalition formation. Exceptions are Jeppesen and Andersen (1998) and Hoel and Schneider (1997), who introduce a non-material payoff from membership or a disutility from breaking the agreement, respectively, and Bosello et al. (2001) who study the stability of coalitions for different equity rules that determine the burden-sharing between cooperating countries. Lange and Vogt (2002) have a different approach on fairness: They rely on a preference structure given by ERCtheory (Bolton and Ockenfels 2000) in which which the utility of a country is not solely based on the absolute payoff but also on the relative payoff compared to the overall 
payoff to all agents. Lange and Vogt (200) show that for countries which are sufficiently inequality-averse with respect to comparing their payoff to those of others, even the grand coalition can be stable. The analysis, however, is restricted to symmetric countries.

This paper extends the analysis of equity-preferences to the heterogeneous country case. According to the U.N. differentiation into developed and developing nations, we assume throughout the paper that only developed countries take on obligations to reduce emissions. Developing countries are exempted from immediate reductions but can provide cheap abatement opportunities via mechanisms like CDM. Although being an exogenous assumption, it reflects concerns of these countries that (i) because of their small per capita emission levels, they are not responsible for the problem, and (ii) a restriction in their emissions would hinder growth and fix their per capita income at a small level. It is widely recognized that developing countries must face emission targets in the long run. For the one-period model considered in this paper, however, the exogenous assumption is reasonable. Throughout the paper we assume that emissions trading among cooperating countries is used to minimize abatement costs.

We first study the effect of allowing abatement in developing countries on the incentives of developed nations to cooperate. We find that the inclusion of such abatement possibilities, e.g. via CDM, can reduce the number of cooperating countries and thereby be welfare-decreasing. We then analyze the impact of equity-preferences on the stability of international agreements: Developed countries may be inequality-averse with respect to (i) the difference in per capita emissions between industrialized and developing countries, and (ii) differences in their abatement target to the average of abatement by all other industrialized countries. ${ }^{4}$ In line with Bolton and Ockenfels (2000), a country's utility is therefore determined by its absolute payoff and the (dis)utility from the respective inequalities.

\footnotetext{
${ }^{4}$ In addition to comparing their abatement to the average, countries clearly might also care about inequalities between other countries. However, with its own abatement target each country can influence its own relative position but not potential inequalities among other countries. We therefore include the comparison to the average of other countries only.
} 
For inequality concerns regarding the gap between per-capita emission levels of industrialized and developing countries, our model predicts that such concerns lead to an increased reduction in industrialized countries but do not qualitatively change the incentives to cooperate. This is different if countries compare their abatement targets with that of other industrialized countries and are inequality-averse with respect to potential differences in abatement targets. By relying on numerical simulations, we show that results substantially change if (some) countries are concerned with this equitycriterion. On one hand, the inclusion of developing countries is generally profitable both in terms of participation rates and of emission reduction if countries are at least slightly inequality-averse. On another hand, we find that equity-preferences lead to a higher degree of cooperation - even the grand coalition can be stabilized if countries are sufficiently inequality-averse. With this, we confirm results by Lange and Vogt (2003) for asymmetric countries.

The paper is organized in the following way: After introducing the model (section 2), we discuss the role of allowing countries to undertake abatement projects in developing countries in section 3. Here we rely on standard preferences. We then discuss the role of equity-preferences in section 4 . We first discuss inequality-concerns regarding the difference in per-capita emissions between developing and industrialized countries (section 4.1) and then turn to studying inequality-aversion with respect to abatement targets (section 4.2). The final section - as always - concludes.

\section{The basic model}

We consider $n$ developed countries, indexed by $i=1, \ldots, n$, and the aggregate of developing countries, $i=0$. Each country $i=0, \ldots, n$ is characterized by its abatement cost function $c_{i}\left(e_{i}\right)$ (decreasing, convex) which depends on the emission level $e_{i}$. We assume linear marginal abatement costs $-c_{i}^{\prime}\left(e_{i}\right)=\alpha_{i}-\beta_{i} e_{i}$, i.e.:

$$
c_{i}\left(e_{i}\right)=\frac{1}{2 \beta_{i}}\left(\alpha_{i}-\beta_{i} e_{i}\right)^{2}
$$


Environmental damages $d_{i}(E)$ in the respective countries depend on the sum of all countries' emissions $E=\sum_{i} e_{i}$. Environmental damages are assumed to be linear:

$$
d_{i}(E)=\gamma_{i} E
$$

The payoff to a country is therefore determined by

$$
y_{i}=-c_{i}\left(e_{i}\right)-d_{i}\left(\sum_{j} e_{j}\right)+t_{i}
$$

where $t_{i}$ denotes transfer payments to country $i$.

If a country cooperates with some other countries, we assume that an emissions trading system is established. Given an emissions target, $\bar{e}_{i}$, a country can achieve this emissions target by reducing by itself, buying permits on the emissions trading market at an endogenous emissions price $p$, or by investing in abatement projects in developing countries. The payment balance according to these activities is captured by the transfers $t_{i}$.

The analysis in this paper relies on a preference structure in which (some) developed countries - along with their own absolute payoff - are possibly motivated by equityconcerns. The approach is similar to the ERC-model by Bolton and Ockenfels (2000) and the approach taken by Lange and Vogt (2002) in which equity is based on the relative payoff of an agent. In order to capture the equity arguments most prominently used in the climate debate, we introduce equity-preferences of industrialized countries with respect to (i) differences in per-capita emissions between developing and industrialized countries (egalitarian arguments), and (ii) differences in emission reduction from the status quo between industrialized countries (possessory arguments). We assume that in the status quo, no climate policy is pursued, i.e. status quo emissions $e_{i}^{0}$ in country $i$ are determined by $-c_{i}^{\prime}\left(e_{i}^{0}\right)=0$.

Let the total population size of industrialized countries $(i=1, \ldots, n)$ be denoted by $L_{\mathrm{IND}}$. Similarly, $\bar{E}_{\mathrm{IND}}$ is the emissions target in industrialized countries which may differ from the actual emissions if abatement projects in developing countries are financed. Analogously for developing countries, the population is denoted by $L_{\mathrm{DEV}}$, the emission 
level by $\bar{E}_{\mathrm{DEV}}=\bar{e}_{0}$. Then, the difference between (assigned) per capita emission levels between industrialized countries and developing countries is measured by

$$
\sigma=\bar{E}_{\mathrm{IND}}-\frac{\bar{E}_{\mathrm{DEV}}}{L_{\mathrm{DEV}}} L_{\mathrm{IND}}
$$

and the relative reduction requirement in country $i$ compared to all industrialized countries is given by

$$
\delta_{i}=\left[\frac{e_{i}^{0}-\bar{e}_{i}}{e_{i}^{0}}-\frac{E_{\mathrm{IND}}^{0}-\bar{E}_{\mathrm{IND}}}{E_{\mathrm{IND}}^{0}}\right] e_{i}^{0}
$$

We assume that if an industrialized country (some people inside these countries) has equity-preferences with respect to per-capita emissions, they suffer some disutility from $\sigma>0$ which increases in $\sigma$. Similarly, inequality-aversion with respect to reduction compared to other industrialized countries induces disutility from $\delta \neq 0$.

To put this more formally, the utility of a developed country $i=1, \ldots, n$ is given by:

$$
U_{i}=y_{i}+a_{i} q\left(\delta_{i}\right)+b_{i} r(\sigma)
$$

where $a_{i}, b_{i} \geq 0$, denotes the weight of the respective equity preference. $r(\cdot)$ is differentiable, concave and decreasing for $\sigma>0 . q(\cdot)$ is also differentiable, concave and has its maximum at $\delta_{i}=0\left(q^{\prime}(0)=0\right)$.

In some parts of the paper we assume for illustrational purposes ${ }^{5}$

$$
q\left(\delta_{i}\right)=-\left(\delta_{i}\right)^{2} / 2 \quad r(\sigma)=-\sigma
$$

In the following, we study a two-stage game of international negotiations as introduced by Barrett (1994), Carraro and Siniscalco (1993), and Hoel (1992). In the first stage, countries decide whether or not to join a coalition $S$. Here, each country takes the decisions of the other countries as given. Each country $i$ also anticipates, however, that the emission levels, which are chosen in the second stage, and national welfare $U_{i}(S)$ depend on the coalition $S$, i.e. on whether it does or does not enter the coalition. In

\footnotetext{
${ }^{5}$ The linear specification of $r(\cdot)$ reflects the fact that because of climate policy, the differences in per-capita emissions are not change too much. Hence, the derivative $r^{\prime}(\cdot)$ is more or less constant. The reduction targets, however, can differ substantially across industrialized countries such that, here, a non-linear function seems to be reasonable.
} 
stage 2, countries inside and outside the coalition simultaneously select their abatement levels. The coalition plays Nash against the non-signatory-countries, which simultaneously maximize their individual utility. A coalition is stable if $U_{i}(S) \geq U_{i}(S \backslash i)$ for $i \in S$ and $U_{i}(S) \geq U_{i}(S+i)$ for $i \notin S$.

\section{Exemptions for developing countries}

In this section we study the consequences of allowing cooperating countries to (partially) achieve their emission reduction by financing projects in developing countries, e.g. via project-based mechanisms like CDM. Developing countries do not get absolute emissions targets. This may be due to equity-considerations, e.g. a lack in responsibility for the historical emissions or the need for economic growth; or, simply, because the willingness-to-pay for climate policy is limited due to more urgent developing problems. In our model which essentially captures only one period, these different arguments can be equivalently captured by $\gamma_{0}=0$. Developing countries can, however, provide abatement possibilities to developed countries via project-based mechanisms like CDM.

In order to decompose the effect of exemptions for developing countries from potential equity-preferences of developed countries, we assume in this section that $a_{i}=b_{i}=0$ for all $i=1, \ldots, n$, i.e. no industrialized country is equity-oriented.

First note that for our specification of utility functions (1), (2), non-cooperating developed countries choose

$$
-c_{i}^{\prime}\left(e_{i}\right)=d_{i}^{\prime}(E) \quad e_{i}^{N}=\frac{\alpha_{i}-\gamma_{i}}{\beta_{i}}
$$

If a coalition $S$ is formed, its members take into account the damages they cause in all cooperating countries. The coalition maximizes the total payoff to its members. ${ }^{6}$ We

\footnotetext{
${ }^{6}$ As we assume that emission allowances can be traded, it is always optimal to maximize total payoff. Individual payoffs can be reshuffled by an appropriate assignment of emission targets $\bar{e}_{i}$.
} 
therefore obtain as the optimal emission levels in a coalition $S$ :

$$
-c_{i}^{\prime}\left(e_{i}\right)=\sum_{i \in S} d_{i}^{\prime}(E) \quad e_{i}^{S}=\frac{\alpha_{i}-\sum_{j \in S} \gamma_{j}}{\beta_{i}}
$$

The aggregate emission level $E(S)$ is then determined by

$$
\begin{aligned}
E(S) & =\sum_{i \notin S} e_{i}^{N}+\sum_{i \in S} e_{i}^{S} \\
& =\sum_{i} \frac{\alpha_{i}}{\beta_{i}}-\sum_{i \notin S} \frac{\gamma_{i}}{\beta_{i}}-\sum_{i \in S} \frac{\sum_{j \in S} \gamma_{j}}{\beta_{i}}
\end{aligned}
$$

The payoff to an individual country in the coalition can be adjusted by assigning a certain number $\bar{e}_{i}^{S}$ of permits which are traded at a price equalling marginal abatement costs, $\sigma^{S}=\sum_{j \in S} \gamma_{j}$. Given a coalition $S$, we denote the payoff to a country $i \in S$ is therefore given by

$$
U_{i}(S)=-c_{i}\left(e_{i}^{S}\right)-d_{i}(E(S))+\sigma^{S}\left(\bar{e}_{i}^{S}-e_{i}^{S}\right)
$$

whereas for $i \notin S$ :

$$
U_{i}(S)=-c_{i}\left(e_{i}^{N}\right)-d_{i}(E(S))
$$

Country $i$ has an incentive to enter the coalition $S$ only if $U_{i}(S \cup i) \geq U_{i}(S \backslash i)$. A coalition $S$ can therefore only be sustained by appropriate allocation of emission rights (or respective transfers) if

$$
\Delta(S):=\sum_{i \in S} U_{i}(S)-\sum_{i \in S} U_{i}(S \backslash i) \geq 0
$$

We show in the appendix that

$$
\Delta(S)=-\frac{3}{2} \sum_{i \in S} \frac{\gamma_{i}^{2}}{\beta_{i}}-\frac{1}{2} \sum_{i \in S} \frac{1}{\beta_{i}}\left(\sum_{i \in S} \gamma_{i}\right)^{2}+\sum_{i \in S} \gamma_{i}\left(\sum_{i \in S} \frac{\gamma_{i}}{\beta_{i}}\right)+\sum_{i \in S} \gamma_{i}^{2}\left(\sum_{i \in S} \frac{1}{\beta_{i}}\right)
$$

We are interested in the effect of the existence of country 0 which does not reduce emissions by itself but allows for other countries to finance abatement activities on the incentives to form a coalition. We therefore compare $\Delta(S \cup 0)$ and $\Delta(S \backslash 0)$. Denoting the number of cooperating industrialized countries by $k(S)$, their average marginal 
damages by $\operatorname{EXP}\left(\gamma_{i}, S\right)=\left(\sum_{i \in S \backslash 0} \gamma_{i}\right) / k(S)$, and its variance by $\operatorname{VAR}\left(\gamma_{i}, S\right)$, simple algebra yields:

$$
\begin{aligned}
\Delta(S \cup 0)-\Delta(S \backslash 0) & =-\frac{1}{2 \beta_{0}}\left(\sum_{j \in S} \gamma_{j}\right)^{2}+\sum_{i \in S} \frac{\gamma_{i}^{2}}{\beta_{0}} \\
& =\frac{k(S)}{2 \beta_{0}}\left[2 \operatorname{VAR}\left(\gamma_{i}, S\right)-(k(S)-2) \operatorname{EXP}\left(\gamma_{i}, S\right)^{2}\right]
\end{aligned}
$$

We obtain the following proposition:

Proposition 1 The incentives to form a particular coalition $S$ of size $k(S)$ can only be increased by allowing for abatement in developing countries if

$$
\operatorname{VAR}\left(\gamma_{i}, S\right)>\frac{k(S)-2}{2} \operatorname{EXP}\left(\gamma_{i}, S\right)^{2}
$$

Therefore, only if the cooperating countries differ sufficiently with respect to their vulnerability from climate change, could the inclusion of developing countries via CDM be used to attract more countries. Otherwise, the possibility of abating in developing countries reduces the incentives to cooperate among developed nations. If countries are similar or, in the extreme, have the same marginal damages $\left(\operatorname{VAR}\left(\gamma_{i}, S\right)=0\right)$, allowing for abatement in a country 0 can never increase the resulting coalition size. If, however, $\operatorname{VAR}\left(\gamma_{i}, S\right)>\frac{k(S)-2}{2} \operatorname{EXP}\left(\gamma_{i}, S\right)^{2}$, then the coalition $S$ could be sustained if only $\beta_{0}$ is small enough, i.e. developing countries can provide very inexpensive abatement opportunities.

Further, consider the case in which $\beta_{i}=\beta$ for all $i$. Here, we obtain from (6):

$$
\begin{aligned}
\Delta(S \backslash 0) & =\frac{k(S)}{2 \beta}\left[(2 k(S)-3) \operatorname{VAR}\left(\gamma_{i}, S\right)-(k(S)-3)(k(S)-1) \operatorname{EXP}\left(\gamma_{i}, S\right)^{2}\right] \\
\Delta(S \cup 0) & =\frac{k(S)}{2 \beta}\left[(2 k(S)-1) \operatorname{VAR}\left(\gamma_{i}, S\right)-\left(k(S)^{2}-3 k(S)+1\right) \operatorname{EXP}\left(\gamma_{i}, S\right)^{2}\right]
\end{aligned}
$$

Hence, without allowing for abatement in country 0, a coalition $S \backslash 0$ can be sustained if:

$$
\operatorname{VAR}\left(\gamma_{i}, S\right) \geq \frac{(k(S)-3)(k(S)-1)}{2 k(S)-3} \operatorname{ExP}\left(\gamma_{i}, S\right)^{2}
$$

whereas $S \cup 0$ can be sustained if

$$
\operatorname{VAR}\left(\gamma_{i}, S\right) \geq \frac{k(S)^{2}-3 k(S)+1}{2 k(S)-1} \operatorname{EXP}\left(\gamma_{i}, S\right)^{2}
$$


It can easily be shown that $\frac{(k(S)-3)(k(S)-1)}{2 k(S)-3}<\frac{k(S)^{2}-3 k(S)+1}{2 k(S)-1}$ for $k(S) \geq 2$. That is, if no country has an incentive to leave the coalition $S \cup 0$, also the coalition $S \backslash 0$ can be sustained. We therefore obtain the following result:

Proposition 2 If the slope of marginal abatement costs is identical across countries, allowing for abatement in developing countries (country 0) can never increase the coalition size of developed countries $(k(S))$.

Note that if countries have the same marginal damages $\left(\operatorname{VAR}\left(\gamma_{i}, S\right)=0\right)$, the maximal coalition size is 3 without allowing for abatement in country 0 - as well-known from the literature. Here, using abatement possibilities in developing countries, reduces the number of cooperating countries to 2 !

Using the results on the number of cooperating countries, we now turn to the environmental effectiveness of the resulting coalitions. It is clear that if the entrance of the developing country does not induce other countries to leave, the total emissions decrease due to the abatement of country 0 by $\sum_{i \in S} \gamma_{i} / \beta_{0}$. If, however, the incentives are reduced such that a country $i \geq 1$ leaves the coalition, this country $i$ increases its emissions by $\sum_{j \in S \backslash i} \gamma_{j} / \beta_{i}$, whereas all remaining countries inside the coalition increase their emissions by a total of $\sum_{j \in S \backslash i} \gamma_{i} / \beta_{j}$. The total effect on emissions from country 0 entering and country $i$ leaving is therefore

$$
\sum_{j \in S, j \neq i, 0}\left[\gamma_{j} / \beta_{i}+\gamma_{i} / \beta_{j}-\gamma_{j} / \beta_{0}\right]
$$

which is positive unless the entering country 0 had a sufficiently small $\beta_{0}$. That is, country 0 would be needed to have a very elastic abatement supply function which leads to a large reduction of emissions to compensate for the increase since the marginal damages caused in country $i$ are no longer taken into account. 


\section{Equity-preferences in (some) developed countries}

\subsection{Equity with respect to per-capita emissions}

Let us now consider the case where some industrialized countries experience disutility from the huge differences in per-capita emissions between industrialized and developing countries, i.e. $b_{i}>0$. We still assume $a_{i}=0$ for all $i$.

Here, for our specification of utility functions, non-cooperating developed countries choose

$$
-c_{i}^{\prime}\left(e_{i}\right)=d_{i}^{\prime}(E)-b_{i} r^{\prime}(\sigma) \quad e_{i}^{N}=\frac{\alpha_{i}-\left(\gamma_{i}+b_{i}\right)}{\beta_{i}}
$$

Similarly, the maximization of total payoff by a coalition $S$ under the (exogenous) assumption that the emissions target of the developing country stays at tis status quo level, leads to

$$
-c_{i}^{\prime}\left(e_{i}\right)=\sum_{i \in S} d_{i}^{\prime}(E)-b_{i} r^{\prime}(\sigma) \quad e_{i}^{S}=\frac{\alpha_{i}-\sum_{j \in S}\left(\gamma_{j}+b_{j}\right)}{\beta_{i}}
$$

Comparing these first order conditions with (4) and (5), it is obvious that such an equity orientation has a similar impact to local environmental damages: It increases the willingness-to-pay for emissions reduction. Countries behave identically to having increased marginal damages, $\gamma_{i}+b_{i}$, but no equity-preference. Abatement levels in industrialized countries therefore increase. Qualitatively, however, all the results from the previous section apply:

Corollary 1 If (some) industrialized countries exhibit equity-preferences based on a comparison of per-capita emissions in industrialized and developing countries, the incentives to cooperate do not change qualitatively. In particular, allowing for abatement in developing countries (country 0) can never increase the coalition size of developed countries $(k(S))$ if the slope of marginal abatement costs is identical across countries. In all coalitions, however, the emission reduction is increased due to the equity-preference. 


\subsection{Inequality-aversion with respect abatement targets}

We now turn to the case of equity-orientation in terms of inequality-aversion with respect to abatement targets in industrialized countries, i.e. $a_{i}>0$. For ease of presentation, we assume here, that $b_{i}=0$ for all $i .^{7}$

Let us first consider a non-cooperating country. This maximizes

$$
\max \sum_{\in S}-c_{i}\left(e_{i}\right)-d_{i}(E)+a_{i} q\left(-e_{i}+\frac{\bar{E}_{\mathrm{IND}}}{E_{\mathrm{IND}}^{0}} e_{i}^{0}\right)
$$

which yields

$$
\begin{aligned}
-c_{i}^{\prime}\left(e_{i}\right) & =d_{i}^{\prime}(E)+a_{i} q^{\prime}\left(\delta_{i}\right)\left(1-\frac{e_{i}^{0}}{E_{\mathrm{IND}}^{0}}\right) \\
\alpha_{i}-\beta_{i} e_{i} & =\gamma_{i}+a_{i}\left(e_{i}-\frac{\bar{E}_{\mathrm{IND}}}{E_{\mathrm{IND}}^{0}} e_{i}^{0}\right)\left(1-\frac{e_{i}^{0}}{E_{\mathrm{IND}}^{0}}\right)
\end{aligned}
$$

A coalition $S$ maximizes its joint payoff. We again assume that the (assigned) emission level of the developing countries is exogenously given at its status quo. Developing countries only provide abatement possibilities to developed countries via project-based mechanisms like CDM. The coalition therefore has to solve the following problem where we define for notational simplicity $a_{0}=0$ :

$$
\begin{aligned}
& \max \sum_{\in S}-c_{i}\left(e_{i}\right)-d_{i}(E)+a_{i} q\left(-\bar{e}_{i}+\frac{\bar{E}_{\mathrm{IND}}}{E_{\mathrm{IND}}^{0}} e_{i}^{0}\right) \\
& \text { s.t. } \sum_{i \in S} e_{i}=\sum_{i \in S} \bar{e}_{i}
\end{aligned}
$$

Let us define $\bar{E}_{S}=\sum_{i \in S \backslash 0} \bar{e}_{i}$ as the emission target of industrialized countries inside the coalition. This can partially be acchieved by abating in the developing country 0 if this is included in the coalition. In this case, we would have $\sum_{i \in S} \bar{e}_{i}=\bar{E}_{S}+e_{0}^{0}$.

Solving the above maximization problem and denoting the Lagrange multiplier by $\lambda$, we obtain

$$
\begin{aligned}
& -c_{i}^{\prime}\left(e_{i}\right)=\lambda+\sum_{j \in S} d_{i}^{\prime}(E) \\
& a_{i} q^{\prime}\left(\delta_{i}\right)=\lambda+\sum_{j \in S} a_{j} q^{\prime}\left(\delta_{j}\right) \frac{e_{j}^{0}}{E_{\mathrm{IND}}^{0}} \text { for all } i \in S \backslash 0
\end{aligned}
$$

\footnotetext{
${ }^{7}$ As in the previous section, $b_{i}>0$ would not change the results!
} 
Consequently, for all industrialized countries inside the coalition we have identical $a_{i} q^{\prime}\left(\delta_{i}\right)$. This implies that either all countries inside the coalition abate more or all abate less than the rest of industrialized countries.

We can rewrite the first-order conditions as

$$
\begin{aligned}
a_{i} q^{\prime}\left(\delta_{i}\right) & =a_{j} q^{\prime}\left(\delta_{j}\right) \\
& =a_{j}\left(\bar{e}_{j}-\frac{\bar{E}_{\mathrm{IND}}}{E_{\mathrm{IND}}^{0}} e_{j}^{0}\right) \\
& =\left(\sum_{j \in S \backslash 0} 1 / a_{j}\right)^{-1}\left(E_{S}-\frac{\bar{E}_{\mathrm{IND}}}{E_{\mathrm{IND}}^{0}} E_{S}^{0}\right) \\
\lambda & =a_{i} q^{\prime}\left(\delta_{i}\right)\left(1-\frac{\sum_{j \in S \backslash 0} e_{j}^{0}}{E_{\mathrm{IND}}^{0}}\right)=a_{i} q^{\prime}\left(\delta_{i}\right)\left(1-\frac{E_{S}^{0}}{E_{\mathrm{IND}}^{0}}\right) \\
& =\left(\sum_{j \in S \backslash 0} 1 / a_{j}\right)^{-1}\left(\bar{E}_{S}-\frac{\bar{E}_{\mathrm{IND}}}{E_{\mathrm{IND}}^{0}} E_{S}^{0}\right)\left(1-\frac{E_{S}^{0}}{E_{\mathrm{IND}}^{0}}\right)
\end{aligned}
$$

and arrive at

$$
\begin{aligned}
-c_{i}^{\prime}\left(e_{i}\right) & =\lambda+\sum_{j \in S} d_{i}^{\prime}(E) \\
\alpha_{i}-\beta_{i} e_{i} & =\sum_{j \in S} \gamma_{j}+\left(\sum_{j \in S \backslash 0} 1 / a_{j}\right)^{-1}\left(\bar{E}_{S}-\frac{\bar{E}_{\mathrm{IND}}}{E_{\mathrm{IND}}^{0}} E_{S}^{0}\right)\left(1-\frac{E_{S}^{0}}{E_{\mathrm{IND}}^{0}}\right)
\end{aligned}
$$

for all $i \in S$.

Note that if at least one country puts no weight on equity-issues $\left(a_{i}=0\right.$ for some $i \in S$ ), the emission choice of the coalition does not depend on the equity-weights of other members of the coalition. Only the internal redistribution of abatement burdens will be affected. If all members of the coalition put some weight on equity, i.e. $a_{i}>0$ for all $i \in S$, a further increase of some $a_{i}$ leads to an extension of emissions if the abatement in the coalition is already larger than that of outsiders. Analogously, the emissions of outsiders change with their equity-parameter.

In general, conditions (7) and (8) provide an implicit definition of emission levels resulting in equilibrium. The formula for the aggregate emission level is given in the Appendix. The impact of higher equity-orientation on aggregate emissions, however, 
is ambiguous. To see this, consider a situation in which exactly one country $i$ puts no weight on equity whereas the equity-parameter $a_{j}$ of all other countries $j \neq i$ gets to infinity. Then, the resulting equilibrium depends on whether or not country $i$ is a member of the coalition. If not $(i \notin S)$, it's emission level stays at $\left(\alpha_{i}-\gamma_{i}\right) / \beta_{i}$, i.e. the abatement (in percent) is given by $\gamma_{i} / \alpha_{i}$. This fraction then determines the (average) abatement of all other countries. The impact of equity-orientation on total emissions therefore depends on how damages in country $i$ compare to those in the rest of the world. Differently, if country $i$ is inside the coalition, the abatement of $S$ is not affected by the increasing equity-orientation. Outsiders, however, adjust their abatement towards the level chosen by the coalition.

We can use these specification of the equilibrium to investigate the stability of coalitions. Here, even for our linear-quadratic specification of the utility functions, a general solution of the problem seems untractable. By relying on numerical examples instead, we can however show that most of the results from the previous section change if (some) countries are inequality-averse with respect to abatement targets. We study the specific case of two different types 1,2 of developed countries, the number of each type being 5 , which differ only with respect to their (marginal) damages and with respect to their equity orientation. We assume

$$
n_{1}=n_{2}=5 \quad \alpha=100 \quad \beta=1
$$

In our numerical simulation we vary the damages $\gamma_{i} \in\{0.5,5\}$ and the respective equity-weight, $a_{i} \in\{0,1,2,5\}$. In order to explore the impact of potentially cheap abatement in developing countries, we look at two specifications of their abatement costs, $\beta_{0} \in\{0.1,1\}$.

Analogously to the previous section, we study the stability of a coalition by looking at the difference in utility levels $\Delta(S)$. We again distinguish two scenarios: DEV if abatement in developing countries is possible, i.e. $0 \in S$, and IND if all abatement has to be undertaken in developed countries, i.e. $0 \notin S$.

Table 1 states the maximal coalitions with their respective aggregate emission level and aggregate payoff for which $\Delta(S) \geq 0$. Here, a stable coalition $S$ is maximal if there 


\begin{tabular}{c|cc|c|c|c} 
& a1 & a2 & Coalition & $\begin{array}{c}\text { total } \\
\text { emissions }\end{array}$ & total payoff \\
\hline \hline IND & $\mathbf{0}$ & $\mathbf{0}$ & $\mathrm{k} 1+\mathrm{k} 2=3$ & 920.00 & -46425 \\
& $\mathbf{1}$ & $\mathbf{1}$ & $\mathrm{k} 1+\mathrm{k} 2=2$ & 967.84 & -48510 \\
& $\mathbf{2}$ & $\mathbf{2}$ & $\mathrm{k} 1+\mathrm{k} 2=2$ & 978.02 & -48969 \\
& $\mathbf{5}$ & $\mathbf{5}$ & $\mathrm{GRAND}$ & 500.00 & -37500 \\
& $\mathbf{0}$ & $\mathbf{5}$ & $\mathrm{k} 1=1, \mathrm{k} 2=4$ & 854.09 & -44400 \\
& & & $\mathrm{k} 1=2, \mathrm{k} 2=1$ & 936.36 & -47268 \\
\hline $\mathbf{D E V}\left(\boldsymbol{\beta}_{\mathbf{0}}=\mathbf{1}\right)$ & $\mathbf{0}$ & $\mathbf{0}$ & $\mathrm{k} 1+\mathrm{k} 2=2$ & 930.00 & -46750 \\
& $\mathbf{1}$ & $\mathbf{1}$ & $\mathrm{k} 1+\mathrm{k} 2=5$ & 814.29 & -42418 \\
& $\mathbf{2}$ & $\mathbf{2}$ & $\mathrm{k} 1+\mathrm{k} 2=6$ & 744.57 & -40273 \\
& $\mathbf{5}$ & $\mathbf{5}$ & $\mathrm{k} 1+\mathrm{k} 2=8$ & 585.23 & -37091 \\
& $\mathbf{0}$ & $\mathbf{5}$ & $\mathrm{k} 1=1, \mathrm{k} 2=3$ & 854.35 & -43932 \\
& & & $\mathrm{k} 1=3, \mathrm{k} 2=1$ & 831.08 & -43013 \\
\hline $\mathbf{D E V}\left(\boldsymbol{\beta}_{\mathbf{0}}=\mathbf{0 . 2}\right)$ & $\mathbf{0}$ & $\mathbf{0}$ & $\mathrm{k} 1+\mathrm{k} 2=2$ & 890.00 & -44950 \\
& $\mathbf{1}$ & $\mathbf{1}$ & $\mathrm{k} 1+\mathrm{k} 2=5$ & 730.77 & -38979 \\
& $\mathbf{2}$ & $\mathbf{2}$ & $\mathrm{GRAND}$ & 250.00 & -31250 \\
& $\mathbf{5}$ & $\mathbf{5}$ & $\mathrm{GRAND}$ & 250.00 & -31250 \\
& $\mathbf{0}$ & $\mathbf{5}$ & $\mathrm{k} 1=1, \mathrm{k} 2=3$ & 758.70 & -40211 \\
& & & $\mathrm{k} 1=2, \mathrm{k} 2=1$ & 793.92 & -41265
\end{tabular}

Table 1: Maximal stable coalitions with total emissions $\bar{E}_{I N D}$ and aggregate payoff levels for industrial countries. Results are given for $\gamma_{1}=\gamma_{2}=5, \alpha=100, \beta_{1}=\beta_{2}=1$, for different levels of inequality-aversion and for $\beta_{0} \in\{0.1,1\}$.

is no $\hat{S} \supset S$ for which $\Delta(\hat{S}) \geq 0$. That is, a maximal coalition cannot be enlarged.

Let us first discuss the results for the symmetric case, $\gamma_{1}=\gamma_{2}=5$. Here, if no abatement in developing countries is possible (scenario IND) and countries are not inequality-averse $\left(a_{1}=a_{2}=0\right)$, maximally 3 countries cooperate as is well-known from the literature. For intermediate levels of inequality-aversion $\left(a_{1}=a_{2}=1,2\right)$, only coalitions of two countries are stable. Here, inequality-aversion obviously leads to a more pessimistic prediction on cooperation, both in terms of the number of cooperating countries as well as in terms of total emissions and total payoff. If, however, countries are sufficiently interested in equity $\left(a_{1}=a_{2}=5\right)$, the grand coalition can be sustained. This basically confirms the result by Lange and Vogt (2003) for the symmetric country case. If only type 2 countries are interested in equity $\left(a_{1}=0, a_{2}=5\right)$ a higher level of cooperation can be sustained.

In line with the previous section, the inclusion of developing countries (country 0) into the coalition cannot improve the cooperation for standard preferences $\left(a_{1}=a_{2}=0\right)$. Rather, it destroys the stability of the 3-country coalition. It thereby leads to welfare- 


\begin{tabular}{|c|c|c|c|c|c|}
\hline & a1 & a2 & Coalition & $\begin{array}{c}\text { total } \\
\text { emissions }\end{array}$ & total payoff \\
\hline IND & $\begin{array}{l}0 \\
1 \\
2 \\
5 \\
0 \\
5\end{array}$ & $\begin{array}{l}0 \\
1 \\
2 \\
5 \\
5 \\
\\
0\end{array}$ & $\begin{array}{c}\mathrm{k} 1=2, \mathrm{k} 2=2 \\
\mathrm{k} 1=5, \mathrm{k} 2=1 \\
\mathrm{k} 1=1, \mathrm{k} 2=2 \\
\mathrm{k} 1=5, \mathrm{k} 2=1 \\
\text { GRAND } \\
\text { GRAND } \\
\mathrm{k} 1=1, \mathrm{k} 2=3 \\
\mathrm{k} 1=3, \mathrm{k} 2=2 \\
\mathrm{k} 1=5, \mathrm{k} 2=1 \\
\mathrm{k} 1=5, \mathrm{k} 2=2\end{array}$ & $\begin{array}{l}939.50 \\
935.00 \\
972.52 \\
957.33 \\
725.00 \\
725.00 \\
934.18 \\
938.77 \\
951.36 \\
897.50\end{array}$ & $\begin{array}{l}-26116 \\
-25931 \\
-26853 \\
-26500 \\
-23719 \\
-23719 \\
-26211 \\
-26200 \\
-26379 \\
-25266\end{array}$ \\
\hline $\operatorname{DEV}\left(\beta_{0}=1\right)$ & $\begin{array}{l}2 \\
5 \\
0 \\
5\end{array}$ & $\begin{array}{l}2 \\
5 \\
5 \\
0\end{array}$ & $\begin{array}{c}\mathrm{k} 1=1, \mathrm{k} 2=2 \\
\mathrm{k} 1=5, \mathrm{k} 2=1 \\
\mathrm{k} 1=1, \mathrm{k} 2=4 \\
\mathrm{k} 1=4, \mathrm{k} 2=3 \\
\mathrm{k} 1=5, \mathrm{k} 2=2 \\
\mathrm{k} 1=2, \mathrm{k} 2=5 \\
\mathrm{k} 1=5, \mathrm{k} 2=4 \\
\mathrm{GRAND} \\
\mathrm{k} 1=1, \mathrm{k} 2=3 \\
\mathrm{k} 1=5, \mathrm{k} 2=2 \\
\mathrm{k} 1=2, \mathrm{k} 2=4 \\
\mathrm{k} 1=4, \mathrm{k} 2=3 \\
\mathrm{k} 1=5, \mathrm{k} 2=2\end{array}$ & $\begin{array}{l}941.00 \\
927.50 \\
860.06 \\
842.75 \\
878.73 \\
762.78 \\
759.25 \\
697.50 \\
902.77 \\
863.86 \\
798.19 \\
840.89 \\
885.00\end{array}$ & $\begin{array}{l}-26136 \\
-25753 \\
-24675 \\
-24353 \\
-24848 \\
-23612 \\
-23526 \\
-23341 \\
-25506 \\
-24599 \\
-23920 \\
-24391 \\
-25000\end{array}$ \\
\hline $\operatorname{DEV}\left(\beta_{0}=0.2\right)$ & $\begin{array}{l}0 \\
1 \\
2 \\
5 \\
0 \\
5\end{array}$ & $\begin{array}{l}0 \\
1 \\
2 \\
5 \\
5 \\
0\end{array}$ & $\begin{array}{c}\mathrm{k} 1=4, \mathrm{k} 2=1 \\
\mathrm{k} 1=1, \mathrm{k} 2=5 \\
\mathrm{k} 1=5, \mathrm{k} 2=4 \\
\text { GRAND } \\
\text { GRAND } \\
\mathrm{k} 1=5, \mathrm{k} 2=2 \\
\text { GRAND }\end{array}$ & $\begin{array}{l}909.50 \\
707.41 \\
668.99 \\
587.50 \\
587.50 \\
797.59 \\
587.50\end{array}$ & $\begin{array}{l}-25306 \\
-22386 \\
-22057 \\
-21828 \\
-21828 \\
-23329 \\
-21828\end{array}$ \\
\hline
\end{tabular}

Table 2: Maximal stable coalitions with total emissions $\bar{E}_{I N D}$ and aggregate payoff levels for industrial countries. Results are given for $\gamma_{1}=0.5, \gamma_{2}=5, \alpha=100, \beta_{1}=\beta_{2}=1$ and for different levels of inequality-aversion and for $\beta_{0} \in\{0.1,1\}$. 
losses if abatement in country 0 is not too cheap: For $\beta_{0}=1$, emissions increase by 10 units. The inclusion of country 0 is only welfare-improving if a developing country can provide low-priced abatement opportunities $\left(\beta_{0}=0.2\right)$ which compensate for the increase in emissions due to the drop-out of one other country. This is different, however, if countries are equity-oriented: Differently from the IND scenario, the coalition size can be increased even for moderate levels of $a_{1}=a_{2}$. For example, for $a_{1}=a_{2}=2$ already a coalition of 5 countries can be sustained if $\beta_{0}=\beta_{1}=\beta_{2}$. The chances of cooperation are even increased if abatement in country 0 gets cheaper, i.e. for $\beta_{0}=0.5<\beta_{1}=\beta_{2}$. Here, the grand coalition can be sustained already for an equity-parameter of 2 .

Let us now look at the asymmetric case $\gamma_{1}=0.5$ and $\gamma_{2}=5$. Here, due to different marginal damages in countries, the coalition size is larger than for the symmetric case even without equity-preferences. For scenario IND, all low damage and one high damage country can cooperate $\left(k_{1}=5, k_{2}=1\right)$. As argued in the previous section, the variance in marginal damages increases cooperation levels since low-damage countries can provide their high-damage counterparts with cheap abatement possibilities and, thus, gain from emissions trading. For increasing inequality-aversion, both total emissions and payoff decrease if the coalition structure stays the same or the stability of a coalition is destroyed $\left(k_{1}=k_{2}=2\right.$ is no longer stable for $\left.a_{1}=a_{2}=1\right)$. However, also in this case, equity-preferences can improve cooperation and efficiency as they may provide enough incentives to form the grand coalition $\left(a_{1}=a_{2} \geq 2\right.$. If we turn to scenario DEV, the inclusion of country 0 can again destroy the stability of a coalition $\left(k_{1}=k_{2}=2\right.$ is no longer stable for $\left.a_{1}=a_{2}=0\right)$ and thereby can lead to efficiency losses. For any level of equity-orientation, however, allowing for abatement in country 0 increases cooperation and abatement rates.

Comparing scenario $\left(a_{1}=5, a_{2}=0\right)$ with $\left(a_{1}=0, a_{2}=5\right)$, it appears to be more important that countries with small marginal damages are equity-oriented. Here, for DEV with $\beta_{0}=0.2$, the grand coalition is stable if $a_{2}=5$, whereas only two high damage countries cooperate if $a_{1}=0$. To understand this result let us study the 
grand coalition. In the grand coalition, emission and utility levels are the same as if all countries would not be interested in equity at all as long as there is one country with $a_{i}=0$. Therefore, if a country with $a_{i}=0$ leaves the coalition, the utility changes are the same as if all countries had standard preferences. However, if an equity-oriented leaves the coalition, it adjusts its abatement towards the average abatement by the remaining coalition. The decision of the rest of the coalition, however, does not depend on inequality-aversion of some members as there is at least one inequality-neutral country. Hence, inequality-aversion influences the incentives to form a coalition, i.e. $\Delta(S)$, only via the changed abatement level of the leaving country. In our case, for a low-damage equity-oriented country this change is sufficient to stabilize the grand coalition. If high-damage countries are equity-oriented, the coalition size can be increased only slightly (to $k_{1}=5, k_{2}=2$ ).

We can summarize our findings as follows:

Result 1 If countries are sufficiently interested in equity, the grand coalition is stable.

Result 2 For coalitions which are stable without equity-preferences, the stability can be destroyed if some countries are equity-interested and no abatement is allowed in developing countries. In this case, equity-preferences lead to larger emission and smaller aggregate payoff levels.

Result 3 The inclusion of developing countries into a coalition by allowing for emission reduction in such countries increases coalition size and aggregate abatement level if countries are interested in equity. This holds true even for moderate levels of inequalityaversion. If countries put no weight on inequality, coalition size and efficiency is decreased by allowing for abatement in developing countries.

Result 4 If countries differ with respect to their marginal damages, cooperation and abatement rates are larger if low-damage countries are equity-oriented than if highdamage countries showed the same degree of equity-orientation. 
Note that Result 3 suggests that the inclusion of developing countries is indeed important to sustain a higher level of cooperation. In reality, inequality aversion with respect to abatement targets can well be motivated by political economy arguments: It is potentially easier to "sell" an agreement if the burdens are apparently similar for all countries. If this is true, allowing for abatement in developing countries which themselves have no binding targets may prove essential to the stability of an agreement. This differs substantially from our findings for standard preferences.

Together with Result 1 this confirms previous findings by Lange and Vogt (2003) that equity-orientation may improve upon the chances of cooperation. Result 2 , however, provides an important caveat: If countries are only slightly driven by inequalityaversion, predictions on cooperation rates can be even more pessimistic than with standard preferences. It is here that the inclusion of developing countries is most important!

\section{Conclusion}

In this paper we analyzed the impact of three different notions of equity on the chances of voluntary cooperation between countries. (i) Equity-driven exemptions for developing countries while opening them for emission reduction projects financed by industrialized countries, (ii) equity-concerns with respect to differences in per-capita emissions between developing and developed countries, and (iii) inequality-aversion regarding the abatement targets.

The assumption of exemptions for developing countries from reduction targets but allowing other countries to use their abatement potential resembles the current structure of the Kyoto protocol: Here, developing countries do not have any binding emission targets but are included via project-based mechanisms like CDM which are financed by industrialized countries. Although the inclusion of such abatement possibilities clearly leads to efficiency gains for any given coalition, it does generally not increase the number of cooperating countries. It can even lead to smaller coalition sizes and thereby be 
welfare-decreasing. Similarly, the chances of cooperation do not qualitatively change if some countries are concerned with the wide gap in per-capita emissions between developing and developed countries. Such concerns might, however, increase the abatement effort by such equity-concerned countries. Only if countries are inequality-averse with respect to comparing their abatement targets with that of all other countries, the cooperation rates are generally increased.

The findings in this paper suggest that project-based emission reduction in developing countries may indeed increase the chances of cooperation - although for a different reason than usually assumed. The general wisdom in the policy debate is that such projects decrease the cost of industrialized countries to achieve a given abatement target and therefore increase the chances of accepting such a target. The paper shows, however, that cheap abatement opportunities and the implied increase in the abatement targets of any coalition provides additional free-riding incentives for outsiders and therby might endanger the stability of a coalition. Chances of cooperation are only increased if these free-riding incentives are limited. This is implicitly the case if countries are inequality-averse with respect to abatement effort. Consequently, the exemption of some countries from absolute abatement targets might eventually prove to beneficial as the chances of cooperation of other countries can be increased.

\section{Appendix}

Derivation of $\Delta(S)$ : First note that

$$
\begin{aligned}
\sum_{i \in S} U_{i}(S) & =\sum_{i \in S}\left[-c_{i}\left(e_{i}^{S}\right)-d_{i}(E(S))\right] \\
& =\sum_{i \in S}\left[-\frac{1}{2 \beta_{i}}\left(\sum_{j \in S} \gamma_{j}\right)^{2}-\gamma_{i} E(S)\right]
\end{aligned}
$$

and

$$
\begin{aligned}
\sum_{i \in S} U_{i}(S \backslash i) & =\sum_{i \in S}\left[-c_{i}\left(e_{i}^{N}\right)-d_{i}(E(S \backslash i))\right] \\
& =\sum_{i \in S}\left[-\frac{1}{2 \beta_{i}} \gamma_{i}^{2}-\gamma_{i} E(S \backslash i)\right]
\end{aligned}
$$


We therefore immediately obtain:

$$
\begin{aligned}
\Delta(S) & =\sum_{i \in S} U_{i}(S)-\sum_{i \in S} U_{i}(S \backslash i) \\
& =\sum_{i \in S}\left[\frac{1}{2 \beta_{i}}\left[\gamma_{i}^{2}-\left(\sum_{j \in S} \gamma_{j}\right)^{2}\right]+\gamma_{i}[E(S \backslash i)-E(S)]\right] \\
& =\sum_{i \in S}\left[\frac{1}{2 \beta_{i}}\left[\gamma_{i}^{2}-\left(\sum_{j \in S} \gamma_{j}\right)^{2}\right]+\gamma_{i}\left[\sum_{j \in S} \frac{\gamma_{j}}{\beta_{i}}+\sum_{j \in S} \frac{\gamma_{i}}{\beta_{j}}-2 \frac{\gamma_{i}}{\beta_{i}}\right]\right] \\
& =-\frac{3}{2} \sum_{i \in S} \frac{\gamma_{i}^{2}}{\beta_{i}}-\frac{1}{2} \sum_{i \in S} \frac{1}{\beta_{i}}\left(\sum_{i \in S} \gamma_{i}\right)^{2}+\sum_{i \in S} \gamma_{i}\left(\sum_{i \in S} \frac{\gamma_{i}}{\beta_{i}}\right)+\sum_{i \in S} \gamma_{i}^{2}\left(\sum_{i \in S} \frac{1}{\beta_{i}}\right)
\end{aligned}
$$

Derivation of total emission level: Rearranging (7) and summing over $i \notin S$ we obtain:

$$
\bar{E}_{\mathrm{IND}}-\bar{E}_{S}=\sum_{i \notin S} \frac{\alpha_{i}-\gamma_{i}}{\beta_{i}+a_{i}\left(1-e_{i}^{0} / E_{\mathrm{IND}}^{0}\right)}+\sum_{i \notin S} \frac{a_{i}\left(1-e_{i}^{0} / E_{\mathrm{IND}}^{0}\right)}{\beta_{i}+a_{i}\left(1-e_{i}^{0} / E_{\mathrm{IND}}^{0}\right)} \frac{e_{i}^{0}}{E_{\mathrm{IND}}^{0}} \bar{E}_{\mathrm{IND}}
$$

Similarly, summing over (8) yields

$$
\bar{E}_{S}=\frac{\sum_{i \in S}\left(\alpha_{i} / \beta_{i}\right)-\left(\sum_{i \in S} \gamma_{i}\right)\left(\sum_{i \in S} 1 / \beta_{i}\right)+\left(\sum_{i \in S} 1 / \beta_{i}\right)\left(\sum_{i \in S} 1 / a_{i}\right)^{-1} \frac{E_{S}^{0}}{E_{\mathrm{IND}}^{0}} \bar{E}_{\mathrm{IND}}\left(1-\frac{E_{S}^{0}}{E_{\mathrm{IND}}^{0}}\right)}{1+\left(\sum_{i \in S} 1 / \beta_{i}\right)\left(\sum_{i \in S} 1 / a_{i}\right)^{-1}\left(1-E_{S}^{0} / E_{\mathrm{IND}}^{0}\right)}
$$

Adding and rearranging these two formulas we finally arrive at:

$$
\begin{aligned}
& \bar{E}_{\mathrm{IND}}=\frac{\frac{\sum_{i \in S}\left(\alpha_{i} / \beta_{i}\right)-\left(\sum_{i \in S} \gamma_{i}\right)\left(\sum_{i \in S} 1 / \beta_{i}\right)}{1+\left(\sum_{i \in S} 1 / \beta_{i}\right)\left(\sum_{i \in S} 1 / a_{i}\right)^{-1}\left(1-\frac{E_{S}^{0}}{E_{\mathrm{IND}}^{0}}\right)}+\sum_{i \notin S} \frac{\alpha_{i}-\gamma_{i}}{\beta_{i}+a_{i}\left(1-e_{i}^{0} / E_{\mathrm{IND}}^{0}\right)}}{1-\frac{\left(\sum_{i \in S} 1 / \beta_{i}\right)\left(1-\frac{E_{S}^{0}}{E_{\mathrm{IND}}^{0}}\right)}{\sum_{i \in S} 1 / a_{i}+\left(\sum_{i \in S} 1 / \beta_{i}\right)\left(1-\frac{E_{S}^{0}}{E_{\mathrm{IND}}^{0}}\right)} \frac{E_{S}^{0}}{E_{\mathrm{IND}}^{N}}-\sum_{i \notin S} \frac{a_{i}\left(1-e_{i}^{0} / E_{\mathrm{IND}}^{0}\right)}{\beta_{i}+a_{i}\left(1-e_{i}^{0} / E_{\mathrm{IND}}^{0}\right)} \frac{e_{i}^{0}}{E_{\mathrm{IND}}^{0}}}
\end{aligned}
$$

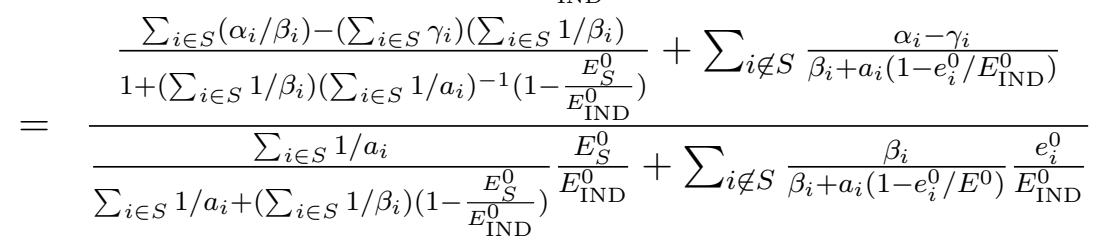

$$
\begin{aligned}
& =E_{\mathrm{IND}}^{0}-\frac{\frac{\left(\sum_{i \in S} \gamma_{i}\right)\left(\sum_{i \in S} 1 / \beta_{i}\right)\left(\sum_{i \in S} 1 / a_{i}\right)}{\left(\sum_{i \in S} 1 / a_{i}\right)+\left(\sum_{i \in S} 1 / \beta_{i}\right)\left(1-\frac{E_{S}^{0}}{E_{\mathrm{IND}}^{0}}\right)}+\sum_{i \notin S} \frac{\gamma_{i}}{\beta_{i}+a_{i}\left(1-e_{i}^{0} / E_{\mathrm{IND}}^{0}\right)}}{\frac{\sum_{i \in S} 1 / a_{i}}{\sum_{i \in S} 1 / a_{i}+\left(\sum_{i \in S} 1 / \beta_{i}\right)\left(1-\frac{E_{S}^{0}}{E_{\mathrm{IND}}^{0}}\right)} \frac{E_{S}^{0}}{E_{\mathrm{IND}}^{0}}+\sum_{i \notin S} \frac{\beta_{i}}{\beta_{i}+a_{i}\left(1-e_{i}^{0} / E_{\mathrm{IND}}^{0}\right)} \frac{e_{i}^{0}}{E_{\mathrm{IND}}^{0}}}
\end{aligned}
$$

where we used that $e_{i}^{0}=\alpha_{i} / \beta_{i}$

For the specific case of two types which differ only with respect to their $a_{i}$ and $\gamma_{i}$ (the developing country having $\beta_{0}$ we obtain for the case of no participation of developing 
countries (scenario IND):

$$
\bar{E}_{\mathrm{IND}}=E_{\mathrm{IND}}^{0}-\frac{\frac{\left(\sum_{i \in S} \gamma_{i}\right) k}{\beta+k\left(\sum_{i \in S} 1 / a_{i}\right)^{-1}(n-k) / n}+\sum_{i \notin S} \frac{\gamma_{i}}{\beta+a_{i}(n-1) / n}}{\frac{\beta}{\beta+\left(\sum_{i \in S} 1 / a_{i}\right)^{-1} k(n-k) / n} \frac{k}{n}+\sum_{i \notin S} \frac{\beta}{\beta+a_{i}(n-1) / n} \frac{1}{n}}
$$

and with participation of the developing country (DEV)

$$
\bar{E}_{\mathrm{IND}}=E_{\mathrm{IND}}^{0}-\frac{\frac{\left(\sum_{i \in S} \gamma_{i}\right)\left(k+\beta / \beta_{0}\right)}{\beta+\left(\sum_{i \in S} 1 / a_{i}\right)^{-1}\left(k+\beta / \beta_{0}\right)(n-k) / n}+\sum_{i \notin S} \frac{\gamma_{i}}{\beta+a_{i}(n-1) / n}}{\frac{\beta}{\beta+\left(\sum_{i \in S} 1 / a_{i}\right)^{-1}\left(k+\beta / \beta_{0}\right)(n-k) / n} \frac{k}{n}+\sum_{i \notin S} \frac{\beta}{\beta+a_{i}(n-1) / n} \frac{1}{n}}
$$

\section{$7 \quad$ References}

Barrett, S. (1992), International environmental agreements as games, in: Pethig, R.(ed.), Conflicts and cooperation in managing environmental resources, Springer, Berlin, 1137.

Barrett, S. (1994), Self enforcing international environmental agreements, Oxford Economic Papers 46, 878-894.

Barrett, S. (1997), Heterogeneous international environmental agreements, in: Carraro, C. (ed.), International environmental agreements: strategic policy issues, E. Elgar, Cheltenham, 9-25.

Barrett, S. (2001), International cooperation for sale, European Economic Review 45, 1835-1850.

Böhringer, C. and C. Helm (2001), Fair Division with General Equilibrium Effects and International Climate Politics, ZEW Discussion Paper No. 01-67, Mannheim.

Böhringer, C. and C. Vogt (2004, forthcoming), The Dismantling of a Breakthrough: The Kyoto-protocol - Just Symbolic Policy, European Journal of Political Economy.

Bosello, F., B. Buchner, C. Carraro, D. Raggi (2001), Can equity enhance efficiency? Lessons from the Kyoto protocol, Nota di Lavoro 49.2001, FEEM, Venice.

Bolton, G.E. and A. Ockenfels (2000), ERC: a theory of equity, reciprocity, and competition, The American Economic Review 90, 166-193. 
Botteon, M. and C. Carraro (1997), Burden sharing and coalition stability in environmental negotiations with asymmetric countries, in: Carraro, C. (ed.), International environmental agreements: strategic policy issues, E. Elgar, Cheltenham, 26-55.

Carraro, C. and D. Siniscalco (1993), Strategies for the international protection of the environment, Journal of Public Economics 52, 309-328.

Cazorla, M.V. and M.A. Toman (2001), International equity and climate change policy, in: Toman, M.A. (ed.), Climate change economics and policy, Resources for the future, Washington, 235-247.

Hoel, M. (1992), International environmental conventions: the case of uniform reductions of emissions, Environmental and Resource Economics 2, 141-59.

Hoel, M. and K. Schneider (1997), Incentives to participate in an international environmental agreement, Environmental and Resource Economics 9, 153-170.

Jeppesen, T. and P. Andersen (1998), Commitment and fairness in environmental games, in: Hanley, N. and H. Folmer: Game theory and the environment, Edward Elgar, Cheltenham, 65-83.

Lange, A. and C. Vogt (2003), Cooperation in international environmental negotiations due to a preference for equity, Journal of Public Economics 87, 2049-2067.

Murdoch, J.C. and T. Sandler (1997), The voluntary provision of a pure public good: the case of reduced CFCs emissions and the Montreal protocol, Journal of Public Economics 63, 331-349.

Raymond, L. (2003), Private rights in public resources - equity and property allocation in market-based environmental policy, Resources for the Future, Washington, DC

Schelling, T. (1960), The strategy of conflict, Harvard University Press, Cambridge, MA.

Tol, R. (2000), Equitable cost-benefit analysis of climate change, in: Carraro, C.: Efficiency and equity in climate change policy, Kluwer: Dordrecht. 